\title{
MICROSCOPICAL AND SEROLOGICAL STUDIES WITH ULTRASTRUCTURE DESCRIPTION OF SARCOCYSTIS SPECIES IN SHEEP IN ASSIUT
}

\author{
BASEM R. NAGEIB ${ }^{1}$ and HUDA M. KURAA ${ }^{2}$ \\ ${ }^{1,2}$ Parasitology Department in Animal Health Research Institute, Assiut Governorate, Egypt
}

Received: 7 March 2018; Accepted: 22 March 2018

\begin{abstract}
This study was conducted to determine the infection rate of Sarcocystis species in sheep in Assiut. A total of 100 slaughtered sheep examined for Sarcocystis, no macroscopic cysts were observed, 93\% (93/100) were positive by microscopical examination and 28\% (28/100) were positive by using Agar gel diffusion test. The infection rate of Sarcocystis had very high significant differences between microscopic examination and Agar gel diffusion test. High statistical significant effect was found on the infection rate of Sarcocystis in different examined muscles of sheep. The highest infection rate was recorded in oesophagus (71\%), followed by diaphragm $(65 \%)$, tongue $(58 \%)$, skeletal muscles $(53 \%)$ and heart muscles $(43 \%)$. The infection rate of Sarcocystis in males was $95.2 \%$ (40/42) while in females were $91.4 \%$ (53/58) by microscopical examination. Higher infection rate of Sarcocystis were detected in sheep 6 months - 2 years age 95\% (38/40) than that of sheep between 2-4 years old $92.7 \%$ (38/41) and those equal or older than 4 years $89.5 \%$ (17/19). No significant difference between infection and age groups or sex of animals was observed. The use of transmission electron microscope (TEM) allowed the identification of S. tenella and S. arieticanis in sheep in Assiut. Examination of S. tenella cyst wall with TEM showed palisade-like villar protrusions while, examination of $S$. arieticanis cyst wall showed hair-like villar protrusions parallel to cyst wall.
\end{abstract}

Key words: Microscopical - serological -ultrastructure - Sarcocystis species - sheep - Assiut.

\section{INTRODUCTION}

Sarcocystis is one of the most prevalent obligate intracellular protozoan parasites belong to Apicomplexan phylum infecting food livestock such as cattle, sheep and goat (Kia et al., 2011). Sarcocystis spp. require two hosts to complete its life cycle; with the sexual cycle in the intestine of a carnivore as a definitive host and asexual cycle in the tissues of an herbivore as an intermediate hosts (Dubey, 1976 and Fayer, 2004). After ingestion of oocysts by the intermediate host, Sarcocystis spp. undergoes asexual generations in the vascular endothelial cells in all parts of the body with eventual formation of mature sarcocysts in the host's muscles. The definitive host is infected due to ingestion of the Sarcocystis infected muscular tissue, which results in the intestinal generation of oocysts (Fayer et al., 2015).

Sarcocystis are common parasites of a broad range of vertebrates. 189 different species can infect

Corresponding author: Dr. BASEM R. NAGEIB

E-mail address: Basemnageib@gmail.com; Huda5380@yahoo.com

Present address: Parasitology Department in Animal Health Research Institute, Assiut Governorate, Egypt mammals, birds, reptiles, and fish even human (Odening, 1998). Infections are recognized to occur in all parts of the world and farm animals are intermediate hosts for a number of species (Dubey et al., 1989a and Hettiarachchi and Rajapakse, 2008). Sarcocystis species infect skeletal muscle, cardiac muscle, and smooth muscle and are rarely found in other tissues (Fayer, 2004).

Sheep are the intermediate host for four species: $S$. tenella (S. ovicanis) and S. arieticanis that are known as pathogenic parasites, which form muscular microcysts transferred by dogs. S. gigantea ( $S$. ovifelis) and $S$. medusiformis are non-pathogenic and form macrocysts transferred by cats (Tenter, 1995; Heckeroth and Tenter, 1998 and Dubey et al., 2015). Sheep become infected with the parasite via ingesting sporocysts or sometimes sporulated oocysts existed in the food or water (Dubey and Lindsay, 2006). Natural infections by Sarcocystis in domestic sheep have been investigated in various countries throughout the world, with prevalence ranging from 9 to $100 \%$ depending on the detection methodology (Dubey et al., 2015).

The severity of clinical signs in domesticated animals depends on the species of parasite and number of 
digested oocysts. S. tenella is the most pathogenic Sarcocystis species in sheep. It can cause anorexia, fever, decreased weight gain, anemia and has been associated with abortions in ewes. Neurologic signs including encephalomyelitis, muscle weakness, hind limb paresis and ataxia have been seen in naturally infected sheep. After recovery from the acute illness, some sheep may lose their wool. Sudden deaths can also occur without other symptoms. Experimentally, S. arieticanis is also pathogenic but somewhat less so than S. tenella. In the other hand S. medusiformis and $S$. gigantea are nonpathogenic or cause only mild disease (Charleston, 1994 and OIE, 2005).

S. tenella causes significant losses in the life stock industry due to death of the animal or abortion of pregnant ewes during acute sarcocystosis, and reduced weight gain, milk and wool production during chronic sarcocystosis (Kia et al., 2011 and Dubey et al., 2015). S. gigantea and S. medusiformis develop macroscopic cysts in striated muscles of sheep which are considered as causes of economic losses in the sheep industry. The heavily infected sheep meat by macroscopic cysts may be condemned as unfit for human consumption during meat inspection in abattoirs. Economic losses in Spain due to Sarcocystis infection to the Spanish sheep industry have been estimated 20 million Euros annually and an average 4 million sheep culled every year (MartínezNavalon et al., 2012 and Farhang-Pajuh et al., 2014)

Identification of different species of Sarcocystis is based on the structure study of the cyst wall by using light or transmission electron microscope of tissue samples from infected hosts, primarily from the skeletal muscle, tongue, heart, diaphragm, and esophagus (Jehle et al., 2009 and Latif et al., 2015).

Several Serological tests such as enzyme-linked immunosorbent assay (ELISA), indirect fluorescent antibody test (IFAT), complement fixation tests and an agar gel diffusion test has also been used to detect sarcocystosis in animals and humans (Hay and Westwood, 2002; Kalita et al., 2015 and Latif et al., 2015).

The aim of the present study was to determine the microscopical and serological prevalence of natural infection with Sarcocystis in the muscles of different organs (tongue, oesophagus, heart, diaphragm and skeletal muscles) of sheep slaughtered in Assuit Gov., Egypt, with identification of the parasite species by light and electron microscope. In addition studying the influence of age, gender of sheep on the infection rate.

\section{MATERIALS AND METHODS}

Collection of samples: In this study, Blood and muscle samples were collected from 100 sheep (42 male and 58 female) with age ranged from 6 momths to 5 years slaughtered at abattoirs from different locations in Assuit Governorate. Fresh tissue samples of tongue, oesophagus, heart, diaphragm and neck skeletal muscle were collected from each animal in clean separate labeled plastic bags. Samples were labeled separately, kept in icebox and rapidly transported to the lab where refrigerated at $4{ }^{\circ} \mathrm{C}$ for further investigation. Moreover, blood sample were collected from each animal during slaughtering in clean, labeled centrifuge tube and their sera were separated by centrifugation and stored at $-20^{\circ} \mathrm{C}$ until tested.

Macroscopic examination: Each fresh sample was thoroughly examined by naked eye for the presence of macroscopic Sarcocystis sp. cysts (Mahran, 2009).

Microscopic examination: According to (Mahran, 2009 and Latif et al., 2015), using Squash method "the compress method" where small pieces of fresh muscle measuring 2-3 $\mathrm{mm}^{3}$ were cut from each tissue sample and squeezed firmly between two glass slides and examined microscopically at $10 \mathrm{x}$ magnifications.

Transmission electron microscopy (TEM): Specimens of muscles were prefixed in $2.5 \%$ glutaraldehyde solution, diced into $1 \mathrm{~mm}^{3}$, then rinsed three times for 15 min each with $0.1 \mathrm{M}$ phosphate buffer ( $\mathrm{pH}$ 7.4). Post-fixation samples were placed in a cold $1 \%$ aqueous osmium tetroxide for $2 \mathrm{~h}$. After rinsing with phosphate buffer again, the specimens were dehydrated in a graded ethanol series of 50$100 \%$ and then embedded in Epon 812. Semithin sections were sliced with glass knives on ultramicrotome for orientation and selection of representative tissue specimens and stained with $0.5 \%$ Toulidine blue according to Bancroft and stevens (1993). Ultrathin sections of $\left(500-800 \mathrm{~A}^{\circ}\right)$ were done using ultramicrotome then contrasted in uranyl acetate and lead citrate, and examined under a (Jeol, CX11) electron microscope in Electron Microscope unit, Assiut University.

\section{Serological examination:}

Preparation of antigen According to Hettiarachchi and Rajapakse (2008): antigen prepared from macroscopically visible Sarcocystis fusiformis cysts were obtained from the oesophagus of heavily infected buffaloes carcasses at Assuit city Abattoir. They were teased out of the muscle with fine forceps, collected into phosphate buffered saline (PBS) PH 7 and stored at $-20{ }^{\circ} \mathrm{C}$. Twinty sarcocysts were collected into tube and washed with saline to remove attached bovine tissue. Using fine forceps the cysts were macerated in $1 \mathrm{~mL}$ of PBS at room temperature until a cream coloured suspension formed. A drop of the suspension was examined under the light microscope (x40) to confirm presence of bananashaped cystozoites. These cystozoites were 
centrifuged at 3000 r.p.m for $10 \mathrm{~min}$, resuspended in PBS (PH 7), recentrifuged at 3000 r.p.m for $10 \mathrm{~min}$ and the supernatant discarded. The pellet of cystozoites was mixed with about $10 \mathrm{~mL}$ of $50 \%$ isotonic Percoll solution (Percoll® P1644-25ML, Sigma-Aldrich Co, St. Louis, MO 63103 USA) in a conical tube and centrifuged at $2500 \mathrm{rpm}$ for $10 \mathrm{~min}$. Extraction buffer consisting of $200 \mu \mathrm{L} 1 \%$ Triton diluted by PBS (PH 7) (TritonX-100, Sigma-Aldrich Co, St. Louis, MO 63103 USA) in PBS was then added to the eppendorf. The sample was mixed well by a whirly mixer for $5 \mathrm{~min}$, while alternatively placing it in ice to avoid heating. The sample was then centrifuged for $10 \mathrm{~min}$ at $14000 \mathrm{rpm}$ in $4^{\circ} \mathrm{C}$. The resulting supernatant (antigen) was collected into eppendorfs then estimated of protein quantity in the antigen using BioTek $®$ Instruments, Inc. (Center of Medical research in Assiut University) and stored at $20^{\circ} \mathrm{C}$.

Agar gel diffusion test: According to Hay and Westwood (2002) and Al-Taee et al. (2009), Fifteen $\mathrm{mL}$ of $1 \%$ agarose solution (Bioshop $®$ Canada Inc., Burlington, ON. L7L 6A4) in 8\% saline was poured into a Petri dish $9 \mathrm{~cm}$ in diameter. Several wells were prepared in the agarose gel at a distance of $3 \mathrm{~mm}$ between the wells. Undiluted and untreated serum was used in this study. Each well in the gel was filled with the antigen or serum to be tested. The dish was kept in a moist chamber at room temperature for 4 days and the reaction was observed daily. When a distinct precipitin line was found in the gel, the serum was considered to have the antibody against Sarcocystis.

Statistical analysis: Statistical analysis was performed by SPSS (ver. 16, IBM, New York, USA) using $\chi^{2}$ test with $95 \%$ confidence interval, with considering $\mathrm{P}$ value $(\mathrm{P}<0.05)$ as significant difference (Mirzaei and Rezaei , 2016).

\section{RESULTS}

One hundred sheep muscle samples were examined for Sarcocystis spp., no macroscopic cysts were observed in the tissues sample of sheep by macroscopic examination. The infection rate of Sarcocystis spp. was 93\% (93/100) by microscopic examination (Figure 1) and 28\% (28/100) by Agar gel diffusion test (Table 1, Figure 2). The infection rate of Sarcocystis had very high significant differences between microscopic examination and Agar gel diffusion test. The infection rate of Sarcocystis spp. in different muscular tissues of sheep (Table 2), showed very high statistical significant effect on the infection rate $(\chi 2=19.212, p>0.001)$. The highest infection rate was recorded in the oesophagus $(71 \%)$ followed by the diaphragm (65\%), tongue (58\%) and skeletal muscles $(53 \%)$; the lower rate was recorded in the heart muscles (43\%). Concerning the sex, the infection rate of Sarcocystis spp. in male sheep was $95.2 \%$ (40/42), while in female was $91.4 \%$ (53/58) by microscopic examination (Table 3). Infection with Sarcocystis associated with age is shown in table 4, higher infection rate among sheep 6 months - 2 years $95 \%(38 / 40)$ than that of sheep between 2-4 years old $92.7 \%(38 / 41)$ and those equal or older than 4 years $89.5 \%$ (17/19). No significant differences between infection and age groups or sex of animals were observed.

The ultrastructural evaluation of Sarcocystis spp. in sheep was mainly targeted to the cyst walls. The use of TEM allowed the identification of $S$. tenella and $S$. arieticanis in sheep. Examination of $S$. tenella cyst in semithin section showed cyst wall with villar protrusions. Metrocytes were adhered the inner surface of the cyst wall followed by bradyzoites in groups separated from each other's with fine septa (Figure 3). Examination of S. tenella cyst with TEM showed that the cyst wall consisted of palisade-like villar protrusions. The ground substance is located directly under the primary cyst wall. Bradyzoites found in inner side of ground substance. Host mitochondria and host cells found near cyst surface (Figure 3,4). Examination of $S$. arieticanis cyst with TEM showed that the cyst wall consisted of hair-like villar protrusions parallel to cyst wall. The ground substance is located directly under the primary cyst wall. Sections of bradyzoites found in inner side of ground substance contain ampylopectin granules. Host mitochondria found near cyst surface (Figure 5).

Table 1: Comparative efficiency of techniques used for detection of Sarcocystis spp. in sheep.

\begin{tabular}{lccccc}
\hline Technique & No. of examined sheep & No. of positive*** & $\%$ & $\chi^{2}$ & P \\
\hline Light microscopy & 100 & 93 & 93 & 88.4 & $<0.0001$ \\
\hline Agar gel diffusion test & 100 & 28 & 28 & & \\
\hline
\end{tabular}

*** Very high significant differences $(\mathrm{P}<0.0001)$ 
Table 2: Infection rate of Sarcocystis spp. in different organs of sheep using light microscope.

\begin{tabular}{|c|c|c|c|c|c|}
\hline Organ & No. of examined & No. of positive**** & $\%$ & $\chi^{2}$ & $\mathbf{P}$ \\
\hline Oesphagus & 100 & 71 & 71 & & \\
\hline Tongue & 100 & 58 & 58 & & \\
\hline Heart & 100 & 43 & 43 & 19.212 & $<0.001$ \\
\hline Diaphragum & 100 & 65 & 65 & & \\
\hline Skeletal muscle & 100 & 53 & 53 & & \\
\hline
\end{tabular}

*** Very high significant differences $(\mathrm{P}<0.001)$

Table 3: Effect of sex on the infection rate of Sarcocystis spp. in sheep using light microscope.

\begin{tabular}{|c|c|c|c|c|c|}
\hline Sex of sheep & No. of examined sheep & No. of positive & $\%$ & $\chi^{2}$ & $\mathbf{P}$ \\
\hline Female & 58 & 53 & 91.4 & & \\
\hline Male & 42 & 40 & 95.2 & 0.557 & $>0.05$ \\
\hline Total & 100 & 93 & 93 & & \\
\hline
\end{tabular}

Insignificant differences $(\mathrm{P}>0.05)$

Table 4: Effect of age on the infection rate of Sarcocystis spp. in sheep using light microscope.

\begin{tabular}{|c|c|c|c|c|c|}
\hline Age of sheep & No. of examined sheep & No. of positive & $\%$ & $\chi^{2}$ & $\mathbf{P}$ \\
\hline $6 m-2 y$ & 40 & 38 & 95 & \multirow{4}{*}{0.615} & \multirow{4}{*}{$>0.05$} \\
\hline 2 to $<4$ year & 41 & 38 & 92.7 & & \\
\hline$\geq 4$ year & 19 & 17 & 89.5 & & \\
\hline Total & 100 & 93 & 93 & & \\
\hline
\end{tabular}

Insignificant differences $(\mathrm{P}>0.05)$ 


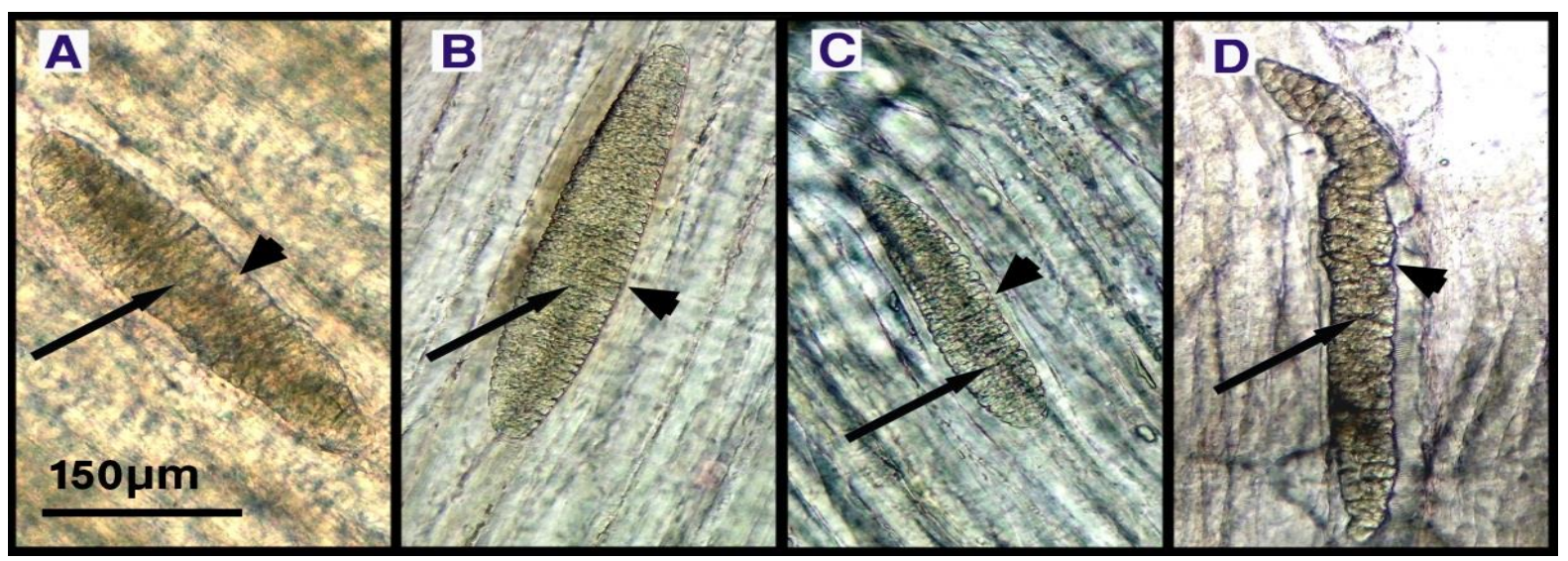

Figure 1: Fresh Sarcocystis spp. cysts in the muscles of sheep compressed between two slides, showing cyst parallel to muscle fibers; Note spindle shaped cyst (arrow head) and septa (arrow) dividing the internal compartments are seen as dark structures (X 10).

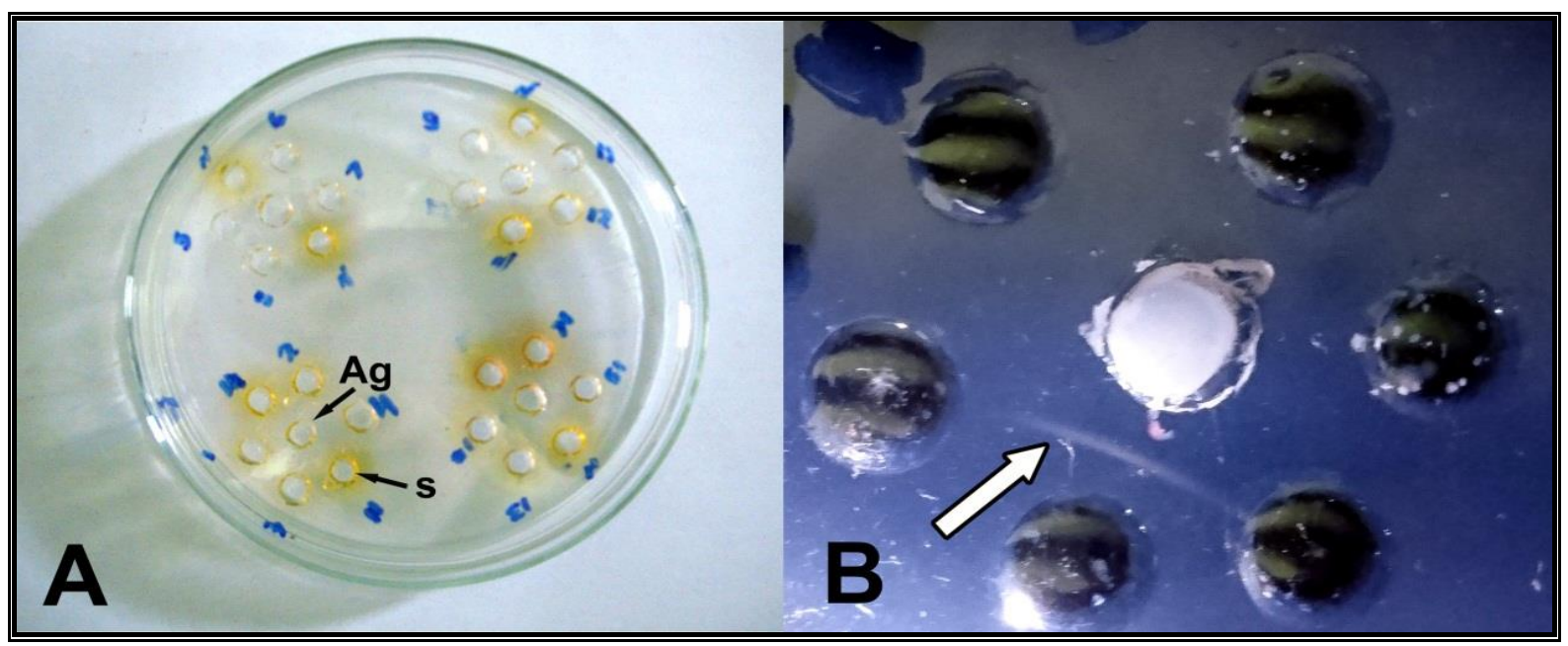

Figure 2: Agar gel diffusion test for diagnosis of Sarcocystis spp. in sheep. A. Agarose gel with Sarcocystis antigen (Ag) in central well and sheep tested sera (S) in peripheral wells in petri dish. B. showing precipitin line (arrow) between positive serum sample and antigen.

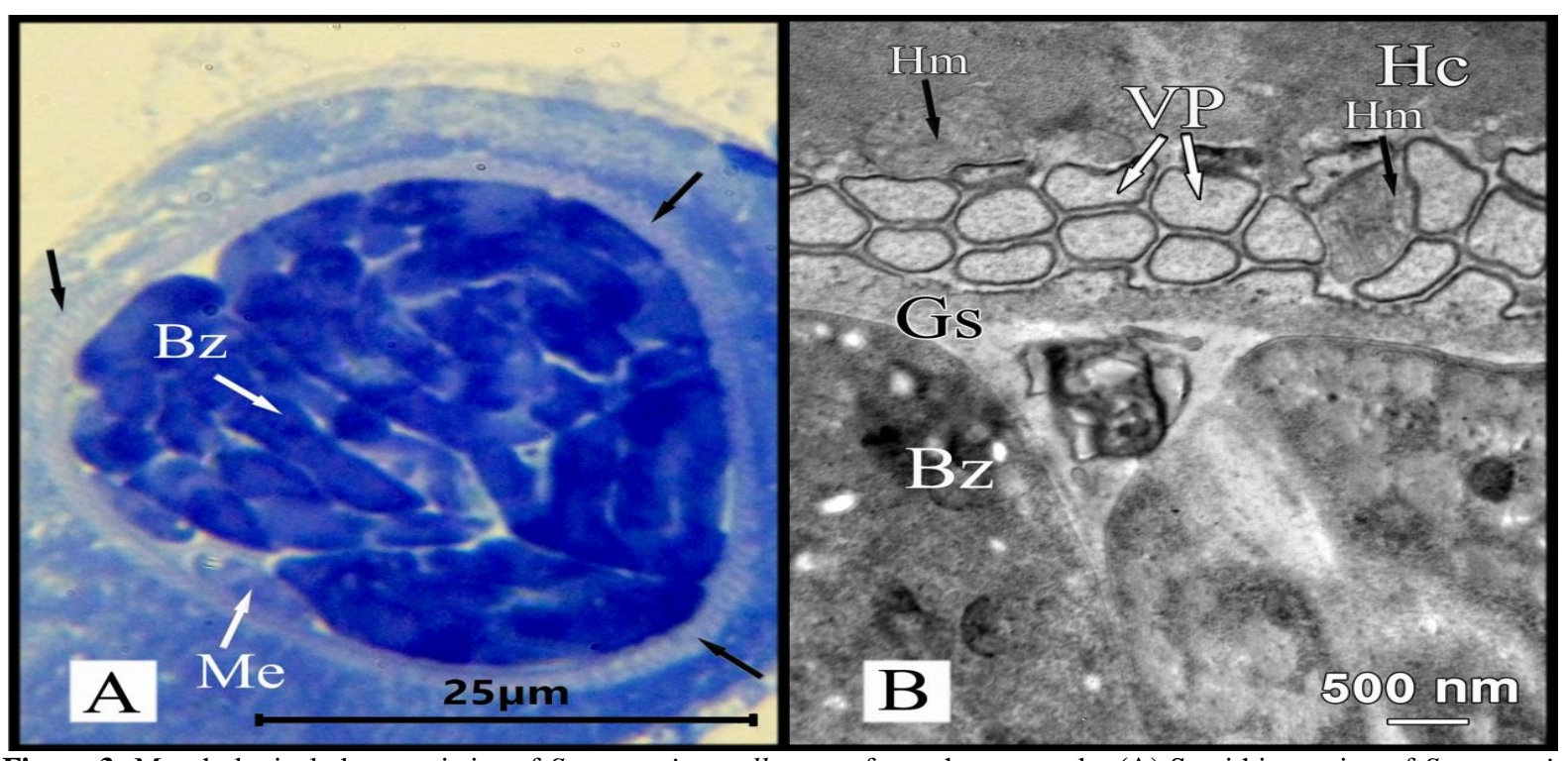

Figure 3: Morphological characteristics of Sarcocystis tenella cysts from sheep muscle. (A) Semithin section of Sarcocystis tenella in muscle of sheep, showing cross section of cyst inside muscle fiber showing villar protrusions (arrows). Metrocytes (Me) found in the inner side of wall followed by bradyzoites (Bz) in chambers. Toluidine blue stain (X100). (B) Transmission electron micrograph (TEM) showing cross-section of a sarcocyst with cross sections of villar protrusions (VP), ground substance $(\mathbf{G s})$ and bradyzoites $(\mathbf{B z})$ in inner side of ground substance. Note host mitochondria $(\mathbf{H m})$ and host cells (Hc) near cyst surface (14000X). 


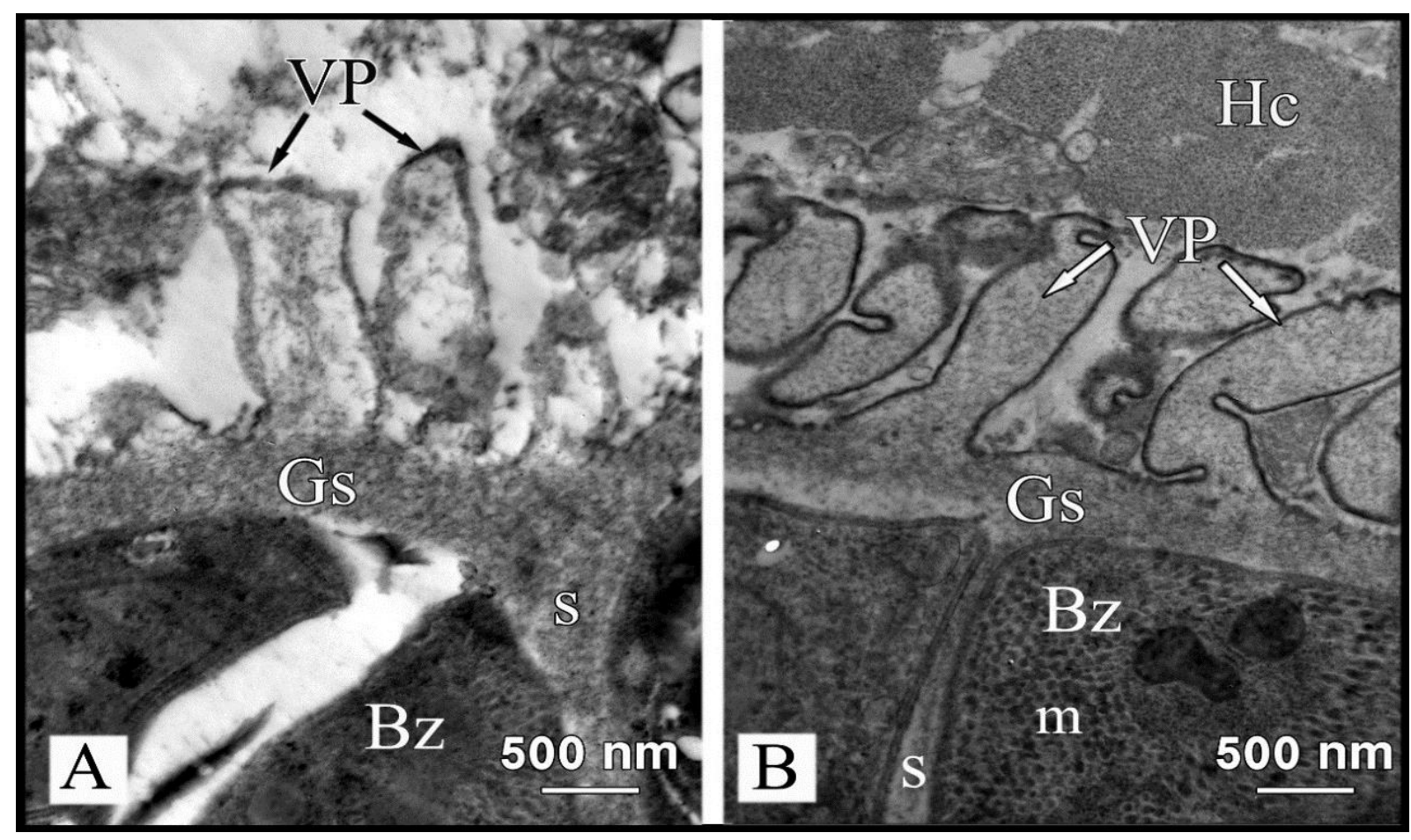

Figure 4: TEM of Sarcocystis tenella from muscle of sheep. (A) TEM showing longitudinal sections showing palisade-like villar protrusions (VP). Sections of bradyzoites (Bz) found inner side of ground substance (Gs) separated by septa (S) (19000X). (B) TEM showing Palisade-like villar protrusions (VP). Sections of bradyzoites $(\mathbf{B z})$ found in inner side of ground substance $(\mathbf{G s})$ contain micronemes $(\mathbf{m})$ separated by septa $(\mathbf{S})$ Note host cell (Hc) near cyst surface (19000X).

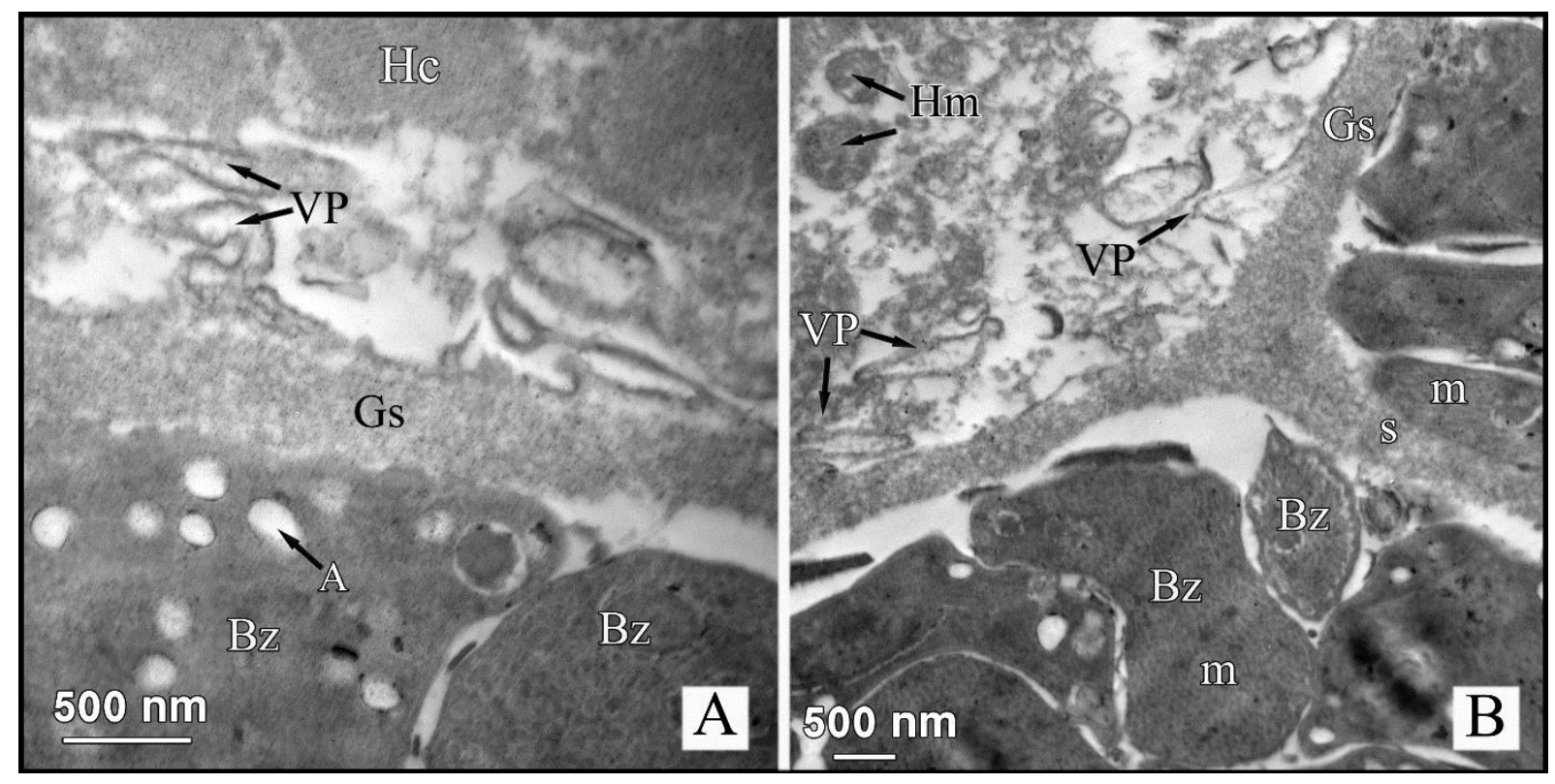

Figure 5: TEM of Sarcocystis arieticanis sarcocysts from sheep muscle. (A) Showing hair-like villar protrusions (VP) parallel to cyst wall. Sections of bradyzoites $(\mathbf{B z})$ found in inner side of ground substance (Gs) contain several ampylopectin granules (A). Note host cell (Hc) near cyst surface (29000X). (B) Showing sections in villar protrusions (VP). Different sections of bradyzoites (Bz) found inside cyst separated by septa (s). Bradyzoites in inner side of ground substance $(\mathbf{G s})$ contain micronemes $(\mathbf{m})$. Note host mitochondria $(\mathbf{H m})$ near cyst surface (14000X). 


\section{DISCUSSION}

Sarcocystis spp. are the most common parasites in domestic ruminants and some of them can generate significant economic losses due to decreased production rates or death of the most severely infected animals (Martínez-Navalón et al., 2012 and Hu et al., 2017).

No macroscopic cysts were observed in the tissues sample of the 100 sheep by macroscopic examination in our study. This result is in line with those recorded before in Dakahlia Governorate, Egypt (Abd ElKader, 2008); Iran (Hajimohammadi et al., 2014); Brazil (Bittencourt et al., 2016) and Iraq (Zangana and Hussein, 2017), whereas Mahran (2009) and ElMishmishy (2017) reported an infection rate of $9.9 \%$ and $0.74 \%$ in sheep in Red Sea and Cairo Governorates, respectively. Moreover, MartínezNavalon et al. (2012) reported an infection rate of $12 \%$ in Spain and (Bahari et al., 2014) 5\% in Iran. The absence of macroscopic sarcocysts in the present study, in comparison with high infection rate of microscopic sarcocysts may be due to the fact that such cysts are of feline origin which characterized by production of low number of sporocysts. The results were in agreement with, Dubey et al. (1989b) who mentioned that Sarcocystis species transmitted by felides have been found less frequent than those transmitted by canids because of low oocyst production in those hosts. Also, Fouly (1997) reported that no sporocysts of Sarcocystis are found in all collected faecal samples of cats in Assiut.

In the present study, the infection rate of Sarcocystis in sheep was 93\% (93/100) by microscopic examination. This result agreed with those recorded by El-Mishmishy (2017) who mentioned that the prevalence of Sarcocystis spp. cysts at 3 Egyptian Provinces (Dakahlia, Damietta and Cairo) was 95.37\%. Moreover studies carried out from different countries have reported similar prevalence of infection in sheep, in Ethiopia (Woldemeskel and Gebreab, 1996) reported an infection rate of $93.6 \%$, also (Bittencourt et al., 2016) and (Hu et al., 2017) reported an infection rates of $95.8 \%$ and $91.9 \%$ in sheep in Brazil and china, respectively. The high infection rate of infection by Sarcocystis in the present work might be due to presence of the examined sheep in rural areas in close association with dogs which contaminate the animal's food with large number of Sarcocystis sporocysts. In addition to that the sporocysts are mostly infective already when passed in the faeces. The result were in agreement with Nourollahi Fard et al. (2009) and Latif et al. (1999) who recorded that carnivorous such as canine, infect environment via faeces by passing 200 million oocyst of Sarcocystis during infection period.

Whereas our result was lower than those previously reported by Whaeeb and Faraj (2016) 100\%, Zangana and Hussein (2017) 97.5\% in Iraq and Hajimohammadi et al. (2014) $97.14 \%$, Mirzaei and Rezaei (2016) $100 \%$ in Iran. On the contrary, lower values were recorded by various authors $(61.41 \%)$ in Assiut Governorate (Taher, 1985); 81.8\% at Shalatin abattoir Red Sea Governorate (Mahran, 2009); $65.15 \%$ in New-Valley Governorate (Elbadre et al., 2014) and from different countries $47.32 \%$ in Turkey (Ozkayhan et al., 2007), 81.5-90\% in K.S.A. (Al Quraishy et al., 2014), 86\% in Malaysia (Latif et al., 2015 ) and $72.2 \%$ in India (Gopal et al., 2016). The variation in these results may be attributed to the difference in the diagnostic technique used, study locality, management conditions and number of samples (Mahran, 2009; Farhang-Pajuh et al., 2014; Latif et al., 2015 and Gopal et al., 2016).

The infection rate of Sarcocystis spp. in sheep was $28 \%$ (28/100) by Agar gel diffusion test. So, using antigen prepared from macroscopically visible Sarcocystis fusiformis cysts could be used in serological diagnosis of sheep Sarcocystis. This finding agrees with that observed by Dubey and Fayer (1983) who recorded that antigen prepared from Sarcocystis of one host generally cross reaction with antibodies against homologous and heterologous species of Sarcocystis. Similar results reported by AlTaee et al. (2009) who revealed that the rate of Sarcocystis sp. infection in 100 sheep was $82 \%$ by Modified Enzyme linked Immunosorbent Assay (ELISA) and $25 \%$ by Agar gel precipitation test in Iraq. Significant differences were detected in sensitivity of these serological tests.

Our results revealed that the infection rate of Sarcocystis in sheep was $93 \%$ by microscopical examination while was $28 \%$ by Agar gel diffusion test. These differences may be due to variation in the intensity of infection in these animals which in turn effect of the amount of the released antibodies that can be detected by Agar gel diffusion test. This finding agree with Gasbarre et al. (1984) who detected that IgG antibody levels of sheep inoculated with Sarcocystis ovicanis was slightly delayed increase (6 to 8 weeks after inoculation was done). Also, measurable IgM antibody response was not seen in sheep.

Concerning the infection rate of Sarcocystis spp. in different muscular tissues of sheep, this study showed variable rates of distribution of sarcocysts in the tissues with very high statistical significant effect on the infection rate $\left(\chi^{2}=19.212, \mathrm{p}>0.001\right)$. The highest infection rate was recorded in the oesophagus $(71 \%)$ followed by the diaphragm $(65 \%)$, tongue $(58 \%)$ and skeletal muscles (53\%); the lower rate was recorded in the heart muscles (43\%). In Red Sea Governorate, Mahran (2009) made similar observation he revealed that maximum sarcocysts were found in the muscles of oesophagus in sheep $(71.1 \%)$ followed the 
diaphragm, tongue, skeletal muscles and the heart muscles $(54.9 \%, 48.6 \%, 40.7 \%$ and $36.9 \%)$, respectively. Likewise, $\mathrm{Hu}$ et al. (2017) in China mentioned that the distribution of the sarcocysts was $84.9 \%$ in esophagus, $32.6 \%$ in tongue, $57 \%$ in diaphragm, $20.9 \%$ in heart and $79.1 \%$ in skeletal muscles. These differences in the infection rate of the organs of sheep might be due to various external factors, such as Sarcocystis spp. responsible for infection, or differences in the study locality and nutritional status of the hosts that may lead to variations in the immunity of the host against infection (Latif et al., 2015).

Observation on the variation due to the sex of sheep on infection rate of sarcocysts infection, showed that males were more infected $95.2 \%$ than females $91.4 \%$, but there was no significant difference between different sexes. This result correlated with the observation of Farhang-Pajuh et al. (2014) that found that the prevalence was $55.17 \%$ and $15.20 \%$ among male and female sheep, respectively. Likewise, Hajimohammadi et al. (2014); Mirzaei and Rezaei (2016) and Zangana and Hussein (2017) found no significant difference between males and females. However, in a study by El-Mishmishy, (2017) he reported that ewes had a higher prevalence of infection $(99 \%)$ than males $(94.55 \%)$. These differences may be due to difference in the management conditions in such localities.

In relation to age; the infection rate of Sarcocystis spp. in sheep showed higher infection rate among sheep 6 monthes- 2 years $(95 \%)$ than that of sheep between 2-4 years old $(92.7 \%)$ and those equal or older than 4 year $(89.5 \%)$, no significant difference was observed. This result agreed with Hajimohammadi et al. (2014) and Mirzaei and Rezaei (2016) that found no significant difference in infection of younger and older animals. While disagreed with Mahran (2009); Elbadre et al. (2014) and El-Mishmishy (2017) that reported higher infection rate among older sheep than that younger one. These differences may be due to difference in location, number of samples and management conditions.

Although light microscopic examination is commonly used for identification of sarcocysts, electron microscopy has been used to identify Sarcocystis spp. according to their wall structure (Haziroglu et al., 2002 and Heckeroth and Tenter, 2007).

In the present study, the use of TEM of Sarcocystis wall showed palisade-like villar protrusions which allowed the identification of Sarcocystis tenella. While, the cyst wall of Sarcocystis that showed hairlike villar protrusions parallel to cyst wall were identified as Sarcocystis arieticanis. These results were in agreement with Dubey et al. (1989b) and Heckeroth and Tenter (2007) who described $S$. arieticanis with hair-like projections in the walls of their cysts. Similar results reported by Bittencourt et al. (2016) and $\mathrm{Hu}$ et al. (2017) who identified the ultrastructural of Sarcocystis tenella and S. arieticanis in tissues from sheep in Brazil and China, respectively.

Our results revealed that $S$. arieticanis cysts were septated, similarly as observed by Heydorn and Mehlhorn (1987) and Bittencourt et al. (2016) who noted that the septa inside some cysts are sometimes not easily visualized, especially in large cysts with large compartments. In contrary, Dubey et al. (1988) described S. arieticanis cysts lack of septa in from sheep in the USA which probably due to the low number of analyzed cysts.

In the present work, TEM revealed the mitochondria were seen to be concentrated near the villar protrusions of Sarcocystis in some host cells of muscle. This finding was similar to that mentioned by Abdel Mawla (1990) who suggested the presence of mitochondria with Sarcocystis infection due to disappearance of myofibrillar pattern in the precystic zone, then loss of the typical linear distribution of the muscle mitochondria and causes their crowding in the ultrastructure study.

Our results revealed a high infection rate of Sarcocystis in sheep. Therefore, we recommend that feed supplies of sheep must be protected from faecal contamination by carnivores. Also, it has of great importance the farmers to be trained not to feed their dogs and cats with uncooked meat in order to be effectively broken of infection cycle between sheep and carnivores.

\section{REFERENCES}

Abd El-Kader, A.I.E. (2008): Studies on tissue parasites of farm animals in Dakahlia Province. Thesis (M.S.) Faculty of Veterinary Medicine, Department of Parasitology- Mansoura University.

Abdel Mawla, M.M. (1990): Ultrastructure of the cyst wall of Sarcocystis lindemanni with pathological correlations. J. Egypt. Soc. Parasitol., 20 (1): 319 - 325.

Al Quraishy, S.; Morsy, K.; Bashtar, A.R.; Ghaffar, F.A. and Mehlhorn, H. (2014): Sarcocystis arieticanis (Apicomplexa: Sarcocystidae) infecting the heart muscles of the domestic sheep, Ovis aries (Artiodactyla: Bovidae), from KSA on the basis of light and electron microscopic data. Parasitology research, 113(10): 3823-3831.

Al-Taee, A.F.; Al-Hyali, N.S. and Al-Badree, M.S. (2009): Seroprevalence of antibodies against Sarcocystis gigantea in different hosts in Ninevah governorate. Iraqi Journal of Veterinary Sciences 23 (1): 107-112. 
Bahari, P.; Salehi, M.; Seyedabadi, M. and Mohammadi, A. (2014): Molecular identification of macroscopic and microscopic cysts of Sarcocystis in sheep in North Khorasan province, Iran. International journal of molecular and cellular medicine, 3(1): 5156.

Bancroft, J.D. and Stevens, A. (1993): Theory and practice of histological techniques. $3^{\text {rd }}$ ed. Churchill Livingstone. Edinburgh, London, Melbourne and New York.

Bittencourt, M.V.; Meneses, I.D.; Ribeiro-Andrade, M.; de Jesus, R.F.; de Araujo, F.R. and Gondim, L.F. (2016): Sarcocystis spp. in sheep and goats: frequency of infection and species identification by morphological, ultrastructural, and molecular tests in Bahia, Brazil. Parasitol Res.; 115(4):1683-1689.

Charleston, W.A.G. (1994): Toxoplasma and other protozoan infections of economic importance in New Zealand. New Zealand journal of zoology, 21(1): 67-81.

Dubey, J.T. (1976): A review of Sarcocystis of domestic animals and of other coccidia of cats and dogs. Journal of the American Veterinary Medical Association, 169(10): 1061-1078.

Dubey, J.P. and Fayer, R. (1983): Sarcocystis. Britich Vet. J., 139; 371-377.

Dubey, J.P. and Lindsay, D.S. (2006): Neosporosis, toxoplasmosis, and sarcocystosis in ruminants. Vet Clin. Food Anim., 22:645-671.

Dubey, J.P.; Lindsay, D.S.; Speer, C.A.; Fayer, R. and Livingston, C.W.Jr. (1988): Sarcocystis arieticanis and other Sarcocystis species in sheep in the United States. J Parasitol 74:1033-1038

Dubey, J.P.; Speer, C.A. and Charleston, W.A.G. (1989a): Ultrastructural differentiation between Sarcocystis of Sarcocystis hirsuta and S. hominis. Vet Parasitol (34):153-157.

Dubey, J.P.; Speer, C.A. and Fayer, R. (1989b): Sarcocystosis of animals and man. CRC Press Inc, Boca Raton, Florida. 105-145.

Dubey, J.P.; Calero-Bernal, R.; Rosenthal, B.M.; Speer, C.A. and Fayer, R. (2015): Sarcocystosis of animals and humans. $2^{\text {nd }}$ edition. CRC Press, Boca Raton, Florida. p 1481.

Elbadre, M.A.; Osman, F.A. and Hussien, S.M. (2014): Studies on Sarcocystis in Sheep and Goats using Serological Survey in New Valley Governorate Egypt. IJAVMS, 8(Issue 5): 150153.

El-Mishmishy, B.M.M. (2017): Molecular characterization of Sarcocystis species in sheep. Thesis (Ph. D.) Faculty of Veterinary Medicine. Department of parasitologyMansoura University.

Farhang-Pajuh, F.; Yakhchali, M. and Mardani, K. (2014): Molecular determination of abundance of infection with Sarcocystis species in slaughtered sheep of Urmia, Iran. Veterinary Research Forum, 5 (3):181-186.

Fayer, R. (2004): Sarcocystis spp. in human infections. Clin Microbiol Rev 17:894-902.

Fayer, R.; Esposito, D.H. and Dubey, J.P. (2015): Human infections with Sarcocystis species. Clin Microbiol Rev 28:295-311.

Fouly, E.A. (1997): Studies on parasites of cats in Assiut Governorate. Ph. D. Thesis, Fac. of Vet. Med., Assiut University.

Gasbarre, L.C.; Suter, P. and Fayer, R. (1984): Humoral and cellular immune responses in cattle and sheep inoculated with Sarcocystis. American Journal of Veterinary Research 45(8):1592-1596.

Gopal, K.; Pazhanivel, N.; Kumar, V. and Thangathurai, R. (2016): Some observations on Sarcocystis Infection in Sheep. Indian Vet. J., 93 (10): 81-82.

Hajimohammadi, B.; Zohourtabar, A.; Fattahi Bafghi, A.; Omidpanah, A.; Rayati, T.; Ghasemi, A. and Sazmand, A. (2014): Occurrence and distribution of Sarcocystis parasite isolated from sheep in Yazd province, Iran. Journal of Community Health Research, 3(3): 205-210.

Hay, F.C. and Westwood, O.M. (2002): Practical Immunology. $4^{\text {th }}$ ed. Black-Well Scientific Publications, London. P 1-400.

Haziroglu, R.; Guvenc, T. and Tunca, R. (2002): Electron microscopical studies on cysts of Sarcocystis arieticanis within cardiac muscle of naturally infected sheep. Parasitology Research 89: 23-25.

Heckeroth, A.R. and Tenter, A.M. (1998): Comparison of immunological and molecular methods for the diagnosis of infections with pathogenic Sarcocystis species in sheep. Tokai J Exp Clin Med.; 23: 293-302.

Heckeroth, A.J. and Tenter, A.M. (2007): Sarcocystiosis. In: Ortega-Mora LM, Gottstein B, Conraths FJ, Buxton D. (eds.): Protozoal Abortion in Farm Ruminants. CAB International, UK. 172-232.

Hettiarachchi, D.C. and Rajapakse, R.P.V.J. (2008): Antigenic analysis of bovine Sarcocystis spp. in Sri Lanka. Journal of the National Science Foundation of Sri Lanka 36 (3):239-244.

Heydorn, A.O. and Mehlhorn, H. (1987): Fine structure of Sarcocystis arieticanis Heydorn, 1985 in its intermediate and final hosts (sheep and dog). Zentralbl Bakteriol Mikrobiol Hyg A 264:353-362

Hu, J-J.; Huang, S.; Wen, T.; Esch, G.W.; Liang, Y. and Li, H-L. (2017): Sarcocystis spp. in domestic sheep in Kunming City, China: prevalence, morphology, and molecular characteristics. Parasite, (24) 30:1-8. 
Jehle, C.; Dinkel, A.; Sander, A.; Morent, M.; Romig, T.; Luc, P.V.; De, T.V.; Thai, V.V. and Mackenstedt, U. (2009): Diagnosis of Sarcocystis spp. in cattle (Bos taurus) and water buffalo (Bubalus bubalis) in Northern Vietnam. Vet Parasitol 23:314-320.

Kalita, M.; Sarmah, P.C. and Singh, S.K. (2015): Evaluation of modified agglutination test in detection of antibodies against Sarcocystis cruzi and Sarcocystis hirsuta of cattle. International Journal of Recent Scientific Research 6, Issue, 8, pp.5968-5970.

Kia, E.B.; Mirhendi, H.; Rezaeian, M.; Zahabiun, F. and Sharbatkhori, M. (2011): First molecular identification of Sarcocystis miescheriana (Protozoa, Apicomplexa) from wild boar (Sus scrofa) in Iran. Experimental parasitology, 127(3): 724-726.

Latif, B.M.A.; Al-Delemi, J.K.; Mohammed, B.S.; AlBayati, S.M. and Amiry, A.M. (1999): Prevalance of Sarcocystis spp. in meat production animals in Iraq. Vet Parasitol; 84(12):85-90.

Latif, B.; Kannan Kutty, M.; Muslim, A.; Hussaini, J.; Omar, E.; Heo, C.C.; Rossle, N.F.; Abdullah, S.; Kamarudin, M.A. and Zulkarnain, M.A. (2015): Light microscopy and molecular identification of Sarcocystis spp. in meat producing animals in Selangor, Malaysia. Tropical Biomedicine 32(3): 444-452.

Mahran, O.M. (2009): Sarcocystis infection in sheep and goats slaughtered in Shalatin Abattoir, Red Sea Governorate, Egypt. Assiut Veterinary Medical Journal, 55(121): 341-355.

Martínez-Navalon, B.; Anastasio-Giner, B.; CanoFructuoso, M.; Sanchez-Martínez, P.; LiopisMorant, A. and Perez-Castarlenas, B., et al. (2012): Sarcocystis infection: a major cause of carcass condemnation in adult sheep in Spain. Spanish J Agric Res., 10(2):388-392.
Mirzaei, M. and Rezaei, H. (2016): The role of sheep in the epidemiology of Sarcocystis spp. in Tabriz area northwest of Iran. J Parasit Dis., 40(2):285-288.

Nourollahi Fard, S.R.; Asghari, M. and Nouri, F. (2009): Survey of Sarcocystis infection in slaughtered cattle in Kerman, Iran. Trop Anim Health Prod; 41(8):1633-1636.

Odening, $K$. (1998): The present state of species systematic in Sarcocystis Lankester, 1882 (Protista, Sporozoa, Coccidie). Systematic Parasitology 41, 209-233.

OIE /Center of Food Security and Public Health (2005): Sarcocystosis. CFSPH, Iowa; 1-6.

Ozkayhan, M.A.; Karaer, Z.; Ilkme, A.N. and Atmaca, H.T. (2007): The prevalence of Sarcocystis species in sheep slaughtered in municipality slaughterhouse in Kirikkale. Türkiye Parazitol. Derg. 31 (4): 272-276.

Taher, G.l.A. (1985): Studies on Sarcocystis of animals in Assiut province. Thesis (M.S.) Faculty of veterinary medicine department of parasitology, Assiut University.

Tenter, A.M. (1995): Current research on Sarcocystis species of domestic animals. Int $\mathrm{J}$ Parasitol 25(11): 1311- 1330

Whaeeb, S.T. and Faraj, A.A. (2016): Molecular Identification and phylogeny of Microscopic Sarcocystis Sheep in Baghdad Province. Int. J. Adv. Res. Biol. Sci. 3(12): 50-56.

Woldemeskel, M. and Gebreab, F. (1996): Prevalence of Sarcocysts in livestock of northwest Ethiopia. Zentralbl Vet Med [B] 43(1):55-58.

Zangana, I.K. and Hussein, S.N. (2017): Prevalence of Sarcocystis Species (Sarcocystis ovicanis and Sarcocystis capricanis) in Tongue Muscle of Sheep and Goats in Duhok Province, Kurdistan Region, North Iraq. Aro-The Scientific Journal of Koya University, 5(1), 3640 .

\section{دراسات مجهرية ومصلية مع توصيف التركيب الدقيق لأنواع الساركوسيست في الأغنام في أسيوط باسم رفعت نجيب ، هلى محدد محد قراعه}

E-mail: Basemnageib@gmail.com; Huda5380@yahoo.com Assiut University web-site: www.aun.edu.eg

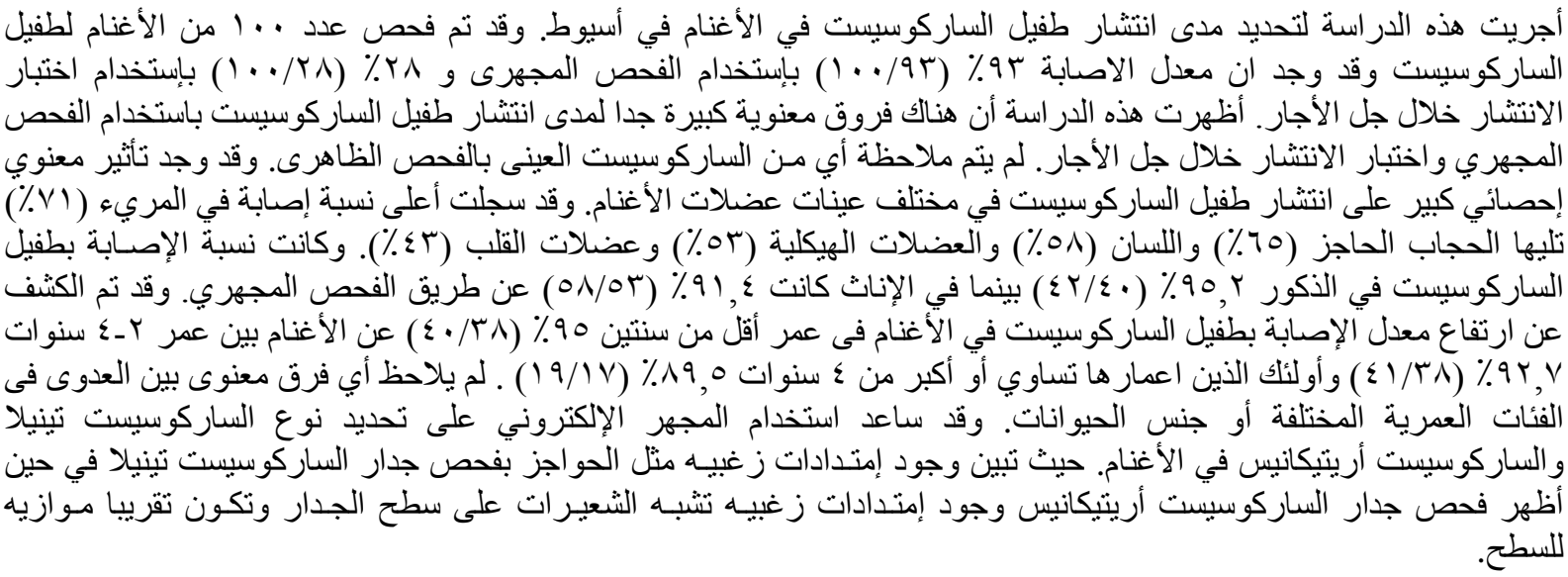

